\title{
CHIRONOMIDAE TYPES IN THE MUSEUM OF ZOOLOGY, BERGEN
}

\author{
Elisabeth Stur \& Trond Andersen \\ Museum of Zoology, University of Bergen, Muséplass 3, N-5007 Bergen, Norway \\ (e-mail: trond.andersen@zmb.uib.no)
}

The International Project Collection at the Museum of Zoology, University of Bergen, contains the holotypes of 239 species of Chironomidae, and paratypes of an additional 133 species, Table 1. More than 1750 slides with type material are housed in the collection. Most of the types belong in the subfamilies
Orthocladiinae and Chironominae, but types of Usambaromyiinae, Podonominae, Tanypodinae, Buchonomyiinae, Diamesinae and Prodiamesinae are also represented.

Inquiries about the Chironomidae in the International Project Collection should be addressed to Trond Andersen.

Table 1. Chironomidae species with types deposited in the International Project Collection at the Museum of Zoology in Bergen.

Abbreviations: $\mathrm{HT}=$ holotype, $\mathrm{PT}=$ Paratype, $\mathrm{m}=$ male, $\mathrm{f}=$ female, $\mathrm{p}=$ pupae, $\mathrm{l}=$ larvae.

\begin{tabular}{|c|c|c|c|c|c|}
\hline $\begin{array}{l}\text { type- } \\
\text { no }\end{array}$ & genus & species / note & author / reference & type & $\begin{array}{l}\text { sex / } \\
\text { stage }\end{array}$ \\
\hline 155 & $\begin{array}{l}\text { USAMBAROMYIINAE } \\
\text { Usambaromyia }\end{array}$ & nigrala & Andersen et Sæther, 1994a & HT, PT & $\mathrm{m}, \mathrm{f}$ \\
\hline & $\begin{array}{l}\text { PODONOMINAE } \\
\text { Lasiodiamesa } \\
\text { Nepodonomus }\end{array}$ & $\begin{array}{l}\text { brusti } \\
\text { similis }\end{array}$ & $\begin{array}{l}\text { Sæther, } 1969 \\
\text { Chaudhuri et Ghosh, } 1981\end{array}$ & $\begin{array}{l}\text { PT } \\
\text { PT }\end{array}$ & $\begin{array}{l}\mathrm{m}, \mathrm{f}, \mathrm{p}, \mathrm{l} \\
\mathrm{m}\end{array}$ \\
\hline & $\begin{array}{l}\text { TANYPODINAE } \\
\text { Ablabesmyia } \\
\text { Clinotanypus } \\
\text { Pentaneurella } \\
\text { Tanypus } \\
\text { Tanypus } \\
\text { Tanypus }\end{array}$ & $\begin{array}{l}\text { maculitibialis } \\
\text { vomerus } \\
\text { katterjokki } \\
\text { grandis } \\
\text { lucidus } \\
\text { tenebrosus }\end{array}$ & $\begin{array}{l}\text { Chaudhuri et al., } 1983 \\
\text { Chaudhuri et Debnath, } 1984 \\
\text { Fittkau et Murray, } 1983 \\
\text { Chaudhuri et al., } 1984 \\
\text { Chaudhuri et al., } 1984 \\
\text { Chaudhuri et al., } 1984\end{array}$ & $\begin{array}{l}\text { PT } \\
\text { PT } \\
\text { PT } \\
\text { PT } \\
\text { PT } \\
\text { PT }\end{array}$ & $\begin{array}{l}\mathrm{m} \\
\mathrm{m} \\
\mathrm{l} \\
\mathrm{m}, \mathrm{f} \\
\mathrm{m} \\
\mathrm{m}\end{array}$ \\
\hline 178 & $\begin{array}{l}\text { BUCHONOMYIINAE } \\
\text { Buchonomyia } \\
\text { Buchonomyia }\end{array}$ & $\begin{array}{l}\text { brundini } \\
\text { burmanica }\end{array}$ & $\begin{array}{l}\text { Andersen et Sæther, 1994b } \\
\text { Brundin et Sæther, } 1978\end{array}$ & $\begin{array}{l}\text { HT, PT } \\
\text { PT }\end{array}$ & $\mathrm{m}$ \\
\hline & $\begin{array}{l}\text { DIAMESINAE } \\
\text { Diamesa } \\
\text { Diamesa }\end{array}$ & $\begin{array}{l}\text { freemani } \\
\text { fonticola }\end{array}$ & $\begin{array}{l}\text { Willassen et Cranston, } 1986 \\
\text { Sæther, } 1969\end{array}$ & $\begin{array}{l}\text { PT } \\
\text { PT }\end{array}$ & $\begin{array}{l}\mathrm{m}, \mathrm{f}, \mathrm{p}, \mathrm{l} \\
\mathrm{f}, \mathrm{p}, \mathrm{l}\end{array}$ \\
\hline 106 & Diamesa & khumbugelida & Sæther et Willassen, 1987 & $\mathrm{HT}, \mathrm{PT}$ & $\mathrm{m}, \mathrm{f}$ \\
\hline 103 & Diamesa & kohshimai & Sæther et Willassen, 1987 & $\mathrm{HT}, \mathrm{PT}$ & $\mathrm{m}, \mathrm{f}, \mathrm{p}$ \\
\hline 83 & Diamesa & lupus & Willassen, 1985 & $\mathrm{HT}, \mathrm{PT}$ & $\mathrm{m}, \mathrm{f}, \mathrm{p}$ \\
\hline 105 & Diamesa & рraесіриа & Sæther et Willassen, 1987 & $\mathrm{HT}, \mathrm{PT}$ & $\mathrm{m}, \mathrm{f}$ \\
\hline 82 & Diamesa & saetheri & Willassen, 1985 & HT, PT & $\mathrm{m}, \mathrm{f}, \mathrm{p}, \mathrm{l}$ \\
\hline 81 & Diamesa & serratosioi & Willassen, 1985 & $\mathrm{HT}, \mathrm{PT}$ & $\mathrm{m}, \mathrm{f}$ \\
\hline 104 & Diamesa & yalavia & Sæther et Willassen, 1987 & HT, PT & $\mathrm{m}, \mathrm{f}, \mathrm{p}$ \\
\hline 114 & $\begin{array}{l}\text { Lappodiamesa } \\
\text { Protanypus } \\
\text { Protanypus } \\
\text { Protanypus }\end{array}$ & $\begin{array}{l}\text { boltoni } \\
\text { hamiltoni } \\
\text { ramosus } \\
\text { saetheri }\end{array}$ & $\begin{array}{l}\text { Sæther et Willassen, } 1988 \\
\text { Sæther, 1975a } \\
\text { Sæther, 1975a } \\
\text { Wiederholm, } 1975\end{array}$ & $\begin{array}{l}\text { HT, PT } \\
\text { PT } \\
\text { PT } \\
\text { PT }\end{array}$ & $\begin{array}{l}\mathrm{m}, \mathrm{f}, \mathrm{p}, \mathrm{l} \\
\mathrm{m}, \mathrm{p}, \mathrm{l} \\
\mathrm{m}, \mathrm{f}, \mathrm{p}, \mathrm{l} \\
\mathrm{m}\end{array}$ \\
\hline 40 & $\begin{array}{l}\text { PRODIAMESINAE } \\
\text { Compteromesa }\end{array}$ & oconeensis & Sæther, 1981a & HT & $\mathrm{m}$ \\
\hline
\end{tabular}




$\begin{aligned} 244 & \begin{array}{l}\text { Monodiamesa } \\ \text { Monodiamesa } \\ \text { Monodiamesa } \\ \text { Monodiamesa }\end{array} \\ 100 & \text { Odontomesa } \\ 99 & \text { Odontomesa }\end{aligned}$

ORTHOCLADIINAE

$\begin{aligned} & \text { Acamptocladius } \\ 25 & \text { Antillocladius } \\ 45 & \text { Antillocladius } \\ 46 & \text { Antillocladius } \\ & \text { Antillocladius } \\ 301 & \text { Antillocladius }\end{aligned}$

Antillocladius

93 Apometriocnemus

Baeoctenus

Boreosmittia

Botryocladius

Botryocladius

Brillia

Brillia

Brillia

374 Bryophaenocladius

375 Bryophaenocladius

127 Bryophaenocladius

376 Bryophaenocladius

340 Bryophaenocladius

Bryophaenocladius

Bryophaenocladius

341 Bryophaenocladius

51 Bryophaenocladius

377 Bryophaenocladius

351 Bryophaenocladius

342 Bryophaenocladius

Chaetocladius

Chaetocladius

Chaetocladius

Chaetocladius

Chaetocladius

30 Compterosmittia

Compterosmittia

49 Compterosmittia

157 Colosmittia

Cricotopus (Cricotopus)

27 C. (Cricotopus)

C. (Cricotopus)

C. (Cricotopus)

28 C. (Cricotopus)

26 C. (Cricotopus)

C. (Cricotopus)

C. (Cricotopus)

245 Diplosmittia

101 Diplosmittia

246 Diplosmittia

34 Diplosmittia

174 Doitrix

Doitrix depectinata
mariae
prolilobata
tuberculata
ferringtoni
fulva nearctica

Sæther, 1973

Andersen, 1996b

Sæther, 1973

Sæther, 1973

Sæther, 1985b

Sæther, 1985b

Cranston et Sæther, 1982

Sæther, 1981c

Sæther, 1982

Sæther, 1982

Wang et Sæther, 1993a

Andersen et Contreras-Ramos, 1999

Wang et Sæther, 1993a

Sæther, 1985h

Sæther, 1976

Tuiskunen, 1986

in Tuiskunen \& Lindeberg 1986

Cranston et Edward, 1999

Cranston et Edward, 1999

Wang et al., 1994

Oliver et Roussel, 1983

Sæther, 1969

Wang, Sæther et Andersen, 2002

Wang, Sæther et Andersen, 2002

Schnell, 1991

Wang, Sæther et Andersen, 2002

Andersen et Schnell, 2000

Ghosh et Chaudhuri, 1983

Ghosh et Chaudhuri, 1983

Andersen et Schnell, 2000

Sæther, 1982

Wang, Sæther et Andersen, 2002

Willassen, 1996

Andersen et Schnell, 2000

Tuiskunen, 1986

in Tuiskunen \& Lindeberg 1986

Cranston et Oliver 1988

Tuiskunen, 1986

in Tuiskunen \& Lindeberg 1986

Sæther, 1969

Chaudhuri et Ghosh, 1982

Sæther, 1981c

Sæther, 1982

Wang, 1998

Andersen et Sæther, 1993a

Cranston et Oliver, 1988

Sæther, 1981c

Oliver, 1977

Sæther, 1971

Sæther, 1981c

Sæther, 1981c

Chaudhuri et Ghosh, 1980

Oliver, 1984

Andersen, 1996a

Sæther, 1985e

Andersen, 1996a

Sæther, 1981c

Sæther et Andersen, 1996

Cranston et Oliver, 1988

$\begin{array}{ll}\text { PT } & \mathrm{m}, \mathrm{p}, \mathrm{l} \\ \text { HT, PT } & \mathrm{m}, \mathrm{f} \\ \text { PT } & \mathrm{m} \\ \text { PT } & 1 \\ \text { HT, PT } & \mathrm{m} \\ \text { HT, PT } & \mathrm{m}, \mathrm{p}, \mathrm{l}\end{array}$

$\begin{array}{ll}\text { PT } & \mathrm{m}, \mathrm{p} \\ \text { HT, PT } & \mathrm{m}, \mathrm{f} \\ \text { HT } & \mathrm{m} \\ \text { HT } & \mathrm{m} \\ \text { PT } & \mathrm{m} \\ & \\ \text { HT } & \mathrm{m} \\ \text { HT } & \mathrm{m} \\ \text { HT } & \mathrm{m} \\ \text { PT } & \mathrm{m}, \mathrm{f}\end{array}$

HT, PT

PT p

PT $p$

HT $\mathrm{m}$

PT $\mathrm{m}$

PT $\mathrm{m}$

HT, PT m

HT, PT $\mathrm{m}$

HT, PT m, f, p, 1

HT $m$

HT, PT m

PT $\mathrm{m}$

PT $\quad m$

HT, PT m

HT $m$

HT, PT m

HT $m$

HT m

PT m

PT p, 1

PT m

PT m, f

PT $m$

HT, PT m, f

HT $\mathrm{m}$

PT $\mathrm{m}$

HT m

PT $\mathrm{m}$

HT m

PT m, l

PT $\mathrm{m}, \mathrm{f}$

HT $m$

HT, PT m, f

PT $m$

PT m, f, 1

HT $m$

HT, PT m

HT $m$

HT, PT m, f

HT $m$

PT $\mathrm{m}$ 


\begin{tabular}{|c|c|}
\hline & Doitrix \\
\hline 56 & Doitrix \\
\hline 173 & Doitrix \\
\hline 74 & Doitrix \\
\hline 55 & Doitrix \\
\hline \multirow[t]{6}{*}{39} & Doncricotopus \\
\hline & Eukiefferiella \\
\hline & Eukiefferiella \\
\hline & Eukiefferiella \\
\hline & Euryhapsis \\
\hline & Euryhapsis \\
\hline 219 & Georthocladius \\
\hline 72 & Georthocladius \\
\hline 75 & Georthocladius \\
\hline \multirow[t]{2}{*}{220} & Georthocladius \\
\hline & Georthocladius \\
\hline 57 & Georthocladius \\
\hline \multirow[t]{2}{*}{79} & Gymnometriocnemus \\
\hline & Hanocladius \\
\hline 166 & Heleniella \\
\hline \multirow{3}{*}{92} & Heleniella \\
\hline & Heterotanytarsus \\
\hline & Heterotanytarsus \\
\hline 129 & Heterotrissocladius \\
\hline \multirow[t]{8}{*}{115} & Heterotrissocladius \\
\hline & Heterotrissocladius \\
\hline & Heterotrissocladius \\
\hline & Heterotrissocladius \\
\hline & Heterotrissocladius \\
\hline & Heterotrissocladius \\
\hline & Hydrobaenus \\
\hline & Habrobaenus \\
\hline \multirow[t]{5}{*}{122} & Hydrobaenus \\
\hline & Hydrobaenus \\
\hline & Hydrobaenus \\
\hline & Hydrobaenus \\
\hline & Hydrobaenus \\
\hline \multirow[t]{2}{*}{121} & Hydrobaenus \\
\hline & Hydrobaenus \\
\hline 158 & Ionthosmittia \\
\hline 154 & Lerheimia \\
\hline 152 & Lerheimia \\
\hline 153 & Lerheimia \\
\hline 123 & Limnophyes \\
\hline 112 & Limnophyes \\
\hline 119 & Limnophyes \\
\hline \multirow[t]{3}{*}{113} & Limnophyes \\
\hline & Limnophyes \\
\hline & Limnophyes \\
\hline 108 & Limnophyes \\
\hline \multirow[t]{2}{*}{109} & Limnophyes \\
\hline & Limnophyes \\
\hline \multirow[t]{5}{*}{126} & Limnophyes \\
\hline & Limnophyes \\
\hline & Limnophyes \\
\hline & Limnophyes \\
\hline & Limnophyes \\
\hline \multirow[t]{4}{*}{120} & Limnophyes \\
\hline & Limnophyes \\
\hline & Limnophyes \\
\hline & Limnophyes \\
\hline
\end{tabular}

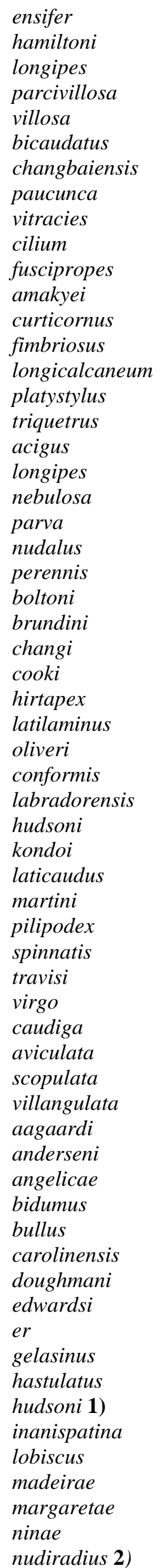

Sæther et Sublette, 1983

Sæther et Sublette, 1983

Sæther et Andersen, 1996

Sæther et Sublette, 1983

Sæther et Sublette, 1983

Sæther, 1981b

Wang et Halvorsen 2002

Sæther, 1969

Sæther, 1969

Oliver, 1981

Sæther et Wang, 1992

Sæther et Andersen, 1996

Sæther et Sublette, 1983

Sæther et Sublette, 1983

Sæther et Andersen, 1996

Sæther et Sublette, 1983

Sæther et Sublette, 1983

Sæther, 1983c

Sæther et Wang 2002

Andersen et Wang, 1997

Sæther, 1985i

Sæther, 1975b

Sæther, 1975b

Sæther, 1992c

Sæther et Schnell, 1988b

Sæther, 1975c

Sæther, 1975c

Sæther, 1975c

Sæther, 1975c

Sæther, 1975c

Sæther, 1976

Sæther, 1977b

Sæther, 1989b

Sæther, 1976

Sæther, 1976

Sæther, 1976

Sæther, 1976

Sæther, 1989b

Sæther, 1976

Sæther et Andersen, 1995

Andersen et Sæther, 1993b

Andersen et Sæther, 1993b

Andersen et Sæther, 1993b

Sæther, 1990

Sæther, 1990

Sæther, 1990

Sæther, 1990

Wang et Sæther, 1993b

Sæther, 1990

Sæther, 1990

Sæther, 1990

Sæther, 1985c

Sæther, 1990

Sæther, 1975d

Sæther, 1975d

Langton et Moubayed, 2001

Sæther, 1990

Sæther, 1990

Sæther, 1975d

Sæther, 1975d

Sæther, 1975d
PT

$\begin{array}{ll}\text { HT, PT } & \mathrm{m} \\ \text { HT } & \mathrm{m}\end{array}$

HT, PT m, p, 1

HT, PT m, p, 1

HT m, p, l

PT $m, f$

PT $\quad \mathrm{m}, \mathrm{p}$

PT $\quad m$

PT $\quad \mathrm{m}, \mathrm{f}$

PT $\mathrm{m}$

HT $m$

HT, PT f, p, 1

HT m, l

HT, PT m

PT $\mathrm{m}$

HT $m$

HT m, f, p

PT $m$

HT m, f

HT, PT m

PT $\quad \mathrm{m}, \mathrm{f}, \mathrm{p}$

PT m, p, 1

HT, PT m, f, p, 1

HT, PT m, f, p, 1

PT m, f, 1

PT $m$

PT m, f, p, 1

PT $\quad f, p, l$

PT $\quad \mathrm{m}, \mathrm{f}, \mathrm{p}, \mathrm{l}$

PT $m, 1$

PT $m$

HT, PT m, f, p, 1

PT $\mathrm{m}$

PT $m$

PT $\mathrm{m}, \mathrm{l}$

PT $m, f$

HT, PT m

PT $\mathrm{f}$

HT, PT m

HT $m$

HT, PT m

HT $m$

HT, PT m

HT, PT m

HT $m$

HT, PT m, f, p

PT $\quad \mathrm{m}, \mathrm{f}$

PT $\mathrm{m}$

HT $m$

HT, PT m, f, p, 1

PT $m$

HT $m$

PT $m$

PT $\mathrm{m}, \mathrm{f}$

HT $m, p$

PT $\mathrm{m}$

HT $m$

PT $\mathrm{m}$

PT $m$ 


\begin{tabular}{|c|c|c|}
\hline & Limnophyes & orbicristatus \\
\hline & Limnophyes & palleocestus \\
\hline & Limnophyes & paludis \\
\hline & Limnophyes & recisus \\
\hline & Limnophyes & roquehautensis \\
\hline \multirow[t]{3}{*}{110} & Limnophyes & schnelli \\
\hline & Limnophyes & spatulosus 3) \\
\hline & Limnophyes & spinigus \\
\hline \multirow[t]{2}{*}{111} & Limnophyes & torulus \\
\hline & Limnophyes & verpus \\
\hline 29 & Lipurometriocnemus & glabalus \\
\hline 47 & Lipurometriocnemus & vixlobatus \\
\hline \multirow[t]{3}{*}{156} & Lobosmittia & basilobata \\
\hline & Lopescladius & fittkaui \\
\hline & Lopescladius & verruculosus \\
\hline 86 & Mesosmittia & acutistylus \\
\hline 355 & Mesosmittia & annae \\
\hline 234 & Mesosmittia & cristaga \\
\hline 357 & Mesosmittia & halata \\
\hline 358 & Mesosmittia & hirta \\
\hline 356 & Mesosmittia & glabra \\
\hline 87 & Mesosmittia & lobiga \\
\hline 88 & Mesosmittia & mina \\
\hline 89 & Mesosmittia & patrihortae \\
\hline 90 & Mesosmittia & prolixa \\
\hline 91 & Mesosmittia & tora \\
\hline 144 & Metriocnemus & acutus \\
\hline 118 & Metriocnemus & brusti \\
\hline \multirow[t]{2}{*}{145} & Metriocnemus & calvescens \\
\hline & Metriocnemus & carmencitabertarum \\
\hline 147 & Metriocnemus & caudigus \\
\hline 145 & Metriocnemus & dentipalpus \\
\hline 149 & Metriocnemus & exilacies \\
\hline 148 & Metriocnemus & intergerivus \\
\hline 143 & Metriocnemus & wangi \\
\hline \multirow[t]{6}{*}{312} & Molleriella & calcarella \\
\hline & Nanocladius & anderseni \\
\hline & Nanocladius & crassicornus \\
\hline & Nanocladius & incomptus \\
\hline & Nanocladius & minimus \\
\hline & Nanocladius & spiniplenus \\
\hline \multirow[t]{4}{*}{102} & Oliveridia & hugginsi \\
\hline & O. (Euorthocladius) & ashei \\
\hline & O. (Euorthocladius) & rousselae \\
\hline & Parachaetocladius & imberbus \\
\hline 38 & Paracricotopus & glaber \\
\hline \multirow[t]{2}{*}{177} & Parakiefferiella & $\operatorname{minax}$ \\
\hline & Parametriocnemus & vespertinus \\
\hline 184 & Paraphaenocladius & crassicaudatus \\
\hline 188 & Paraphaenocladius & exagitans longipes \\
\hline 189 & Paraphaenocladius & impensus contractus \\
\hline 187 & Paraphaenocladius & innasus \\
\hline 182 & Paraphaenocladius & irritus longicostatus \\
\hline 186 & Paraphaenocladius & pusillus \\
\hline 186 & Paraphaenocladius & proprius \\
\hline \multirow[t]{2}{*}{183} & Paraphaenocladius & pseudirritus nearcticus \\
\hline & Phycoidella & dentolatens 4) \\
\hline 279 & Physoneura & paulseni \\
\hline \multirow[t]{3}{*}{53} & Platysmittia & fimbriata \\
\hline & Platysmittia & bilyji \\
\hline & Plhudsonia & acuticauda \\
\hline 44 & Plhudsonia & partita \\
\hline
\end{tabular}

Limnophyes

Limnophyes

Limnophyes

Limnophyes

47 Lipurometriocnemus

opescladius

355

Mesosmittia

6 Mesosmittia

87 Mesosmittia

91 Mesosmittia

144 Metriocnemus

118 Metriocnemus
Wang et Sæther 1993b

Wang et Sæther, 1993b

Armitage, 1985

Sæther, 1975d

Langton et Moubayed, 2001

Sæther, 1990

Sæther, 1975d

Sæther, 1990

Sæther, 1990

Wang et Sæther, 1993b

Sæther, 1981c

Sæther, 1982

Sæther et Andersen, 1993

Sæther, 1983b

Sæther, 1983b

Sæther, 1985d

Andersen et Mendes, 2002b

Sæther, 1996

Andersen et Mendes, 2002b

Andersen et Mendes, 2002b

Andersen et Mendes, 2002b

Sæther, 1985d

Sæther, 1985d

Sæther, 1985d

Sæther, 1985d

Sæther, 1985d

Sæther, 1995

Sæther, 1989a

Sæther, 1995

Langton et Cobo, 1997

Sæther, 1995

Sæther, 1995

Sæther, 1995

Sæther, 1995

Sæther, 1995

Sæther et Ekrem, 1999

Sæther, 1977a

Sæther, 1977a

Sæther, 1977a

Sæther, 1977a

Sæther, 1977a

Ferrington et Sæther, 1987

Soponis, 1990

Soponis, 1990

Sæther et Sublette, 1983

Sæther, 1980

Ferrington et Sæther, 1994

Sæther, 1969

Sæther et Wang, 1995

Sæther et Wang, 1995

Sæther et Wang, 1995

Sæther et Wang, 1995

Sæther et Wang, 1995

Sæther et Wang, 1995

Chaudhuri et Sinharay, 1987

Sæther et Wang, 1995

Sæther, 1971

Stur et Andersen, 2000

Sæther, 1982

Sæther, 1985g

Sæther, 1992a

Sæther, 1982

\begin{tabular}{|c|c|}
\hline $\mathrm{PT}$ & $\mathrm{m}$ \\
\hline PT & $\mathrm{m}$ \\
\hline PT & $\mathrm{m}$ \\
\hline PT & $\mathrm{m}$ \\
\hline HT & $\mathrm{m}$ \\
\hline HT, PT & $\mathrm{m}$ \\
\hline PT & $\mathrm{m}$ \\
\hline PT & $\mathrm{m}$ \\
\hline HT & $\mathrm{m}$ \\
\hline PT & $\mathrm{m}$ \\
\hline HT, PT & $\mathrm{m}, \mathrm{f}$ \\
\hline HT & $\mathrm{m}$ \\
\hline HT & $\mathrm{m}$ \\
\hline PT & $\mathrm{m}, \mathrm{p}$ \\
\hline PT & $\mathrm{p}$ \\
\hline HT & $\mathrm{m}$ \\
\hline HT, PT & $\mathrm{m}$ \\
\hline $\mathrm{HT}$ & $\mathrm{m}$ \\
\hline HT, PT & $\mathrm{m}$ \\
\hline HT & $\mathrm{m}$ \\
\hline HT, PT & $\mathrm{m}$ \\
\hline $\mathrm{HT}$ & $\mathrm{m}$ \\
\hline $\mathrm{HT}$ & $\mathrm{m}$ \\
\hline HT, PT & $\mathrm{m}$ \\
\hline HT, PT & $\mathrm{m}$ \\
\hline $\mathrm{HT}$ & $\mathrm{m}$ \\
\hline HT & $\mathrm{m}$ \\
\hline $\mathrm{HT}, \mathrm{PT}$ & $\mathrm{m}, \mathrm{p}, 1$ \\
\hline $\mathrm{HT}$ & $\mathrm{m}$ \\
\hline HT, PT & $\mathrm{m}, \mathrm{f}, \mathrm{p}, \mathrm{l}$ \\
\hline HT, PT & $\mathrm{m}$ \\
\hline HT & $\mathrm{m}$ \\
\hline HT, PT & $\mathrm{m}$ \\
\hline HT, PT & $\mathrm{m}, \mathrm{f}$ \\
\hline HT, PT & $\mathrm{m}$ \\
\hline $\mathrm{HT}, \mathrm{PT}$ & $\mathrm{m}, \mathrm{f}$ \\
\hline PT & $\mathrm{m}, \mathrm{f}, \mathrm{p}, \mathrm{l}$ \\
\hline PT & $\mathrm{p}$ \\
\hline PT & $\mathrm{m}, \mathrm{f}$ \\
\hline PT & $\mathrm{m}, \mathrm{p}$ \\
\hline PT & $\mathrm{p}$ \\
\hline HT, PT & $\mathrm{m}, \mathrm{f}, \mathrm{p}$ \\
\hline PT & $\mathrm{m}, \mathrm{p}, 1$ \\
\hline PT & $\mathrm{m}, \mathrm{p}, 1$ \\
\hline PT & $\mathrm{m}$ \\
\hline $\mathrm{HT}, \mathrm{PT}$ & $\mathrm{m}, \mathrm{f}, \mathrm{p}, \mathrm{l}$ \\
\hline HT & $\mathrm{m}$ \\
\hline PT & $\mathrm{m}$ \\
\hline $\mathrm{HT}, \mathrm{PT}$ & $\mathrm{m}$ \\
\hline HT, PT & $\mathrm{m}$ \\
\hline HT, PT & $\mathrm{m}$ \\
\hline $\mathrm{HT}, \mathrm{PT}$ & $\mathrm{m}$ \\
\hline HT & $\mathrm{m}$ \\
\hline HT, PT & $\mathrm{m}$ \\
\hline PT & $\mathrm{m}$ \\
\hline HT, PT & $\mathrm{m}$ \\
\hline PT & 1 \\
\hline HT, PT & $\mathrm{m}$ \\
\hline HT & $\mathrm{m}$ \\
\hline PT & $\mathrm{f}$ \\
\hline PT & $\mathrm{m}, \mathrm{p}$ \\
\hline $\mathrm{HT}, \mathrm{PT}$ & $\mathrm{m}, \mathrm{p}$ \\
\hline
\end{tabular}


Pseudorthocladius

60 Pseudorthocladius

67 Pseudorthocladius

63 Pseudorthocladius

58 Pseudorthocladius

77 Pseudorthocladius

66 Pseudorthocladius

78 Pseudorthocladius

65 Pseudorthocladius

59 Pseudorthocladius

61 Pseudorthocladius

62 Pseudorthocladius

64 Pseudorthocladius

68 Pseudorthocladius

48 Psilometriocnemus Quiniella

97 Rheocricotopus

96 Rheocricotopus

98 Rheocricotopus Rheocricotopus Rheocricotopus Rheocricotopus Rheocricotopus Rheocricotopus

116 Rheocricotopus

117 Rheocricotopus Rheocricotopus $R$. (Psilocricotopus)

95 Rheosmittia

54 Saetheriella

50 Stilocladius

80 Sublettiella

120 Tavastia Thalassosmittia

94 Thienemannia

269 Thienemanniella

270 Thienemanniella

36 Thienemanniella

35 Thienemanniella

347 Thienemanniella Tokunagayusurika

149 Tokyobrillia

52 Unniella

107 Vivacricotopus Xylotopus

Zalutschia

Zalutschia

Zalutschia

Zalutschia

Zalutschia

\section{CHIRONOMINAE}

359 Axarus

170 Beardius

171 Beardius

Beardius

172 Beardius Cyphomella

248 Friederia Harnishia

84 Oschia sinicus

amplicaudus

clavatosus

comans

curticornus

destitutus

lunatus

macrovirgatus

morsei

paravirgatus

rectilobus

tricanthus

uniserratus

virgatus

wingoi

cristatus

lii

amplicristatus

conflusirius

effusoides

eminellobus

himalayensis

kenorensis

nemoacrostichalis

pauciseta

reduncus

unidentatus

valgus

calviculus

halvorseni 5)

amplicristata

clinopecten

calvata

cristacauda

montana

pilinucha

boltoni

lobapodema

sanctivincenta

semifimbriata 6)

taurocapita

taihuensis 7)

anderseni

multivirga

ablusus

burmanensis

lingulata lingulata

lingulata pauca

pusa

trigonacies

vockerothi

\section{Chironomini}

froelichi

aciculatus

lingulatus

parcus

triangulatus

gibbera

villosa

turgidula

dorsenna $\mathbf{8}$ )
Sæther et Wang, 1996

Sæther et Sublette, 1983

Sæther et Sublette, 1983

Sæther et Sublette, 1983

Sæther et Sublette, 1983

Sæther et Sublette, 1983

Sæther et Sublette, 1983

Sæther et Sublette, 1983

Sæther et Sublette, 1983

Sæther et Sublette, 1983

Sæther et Sublette, 1983

Sæther et Sublette, 1983

Sæther et Sublette, 1983

Sæther et Sublette, 1983

Sæther et Sublette, 1983

Sæther, 1982

Wang et Sæther, 1998

Sæther, $1985 f$

Sæther, $1985 f$

Sæther, 1985f

Sæther, 1969

Chaudhuri et Sinharay, 1983

Sæther, 1969

Chaudhuri et Sinharay, 1983

Sæther, 1969

Sæther et Schnell, 1988a

Sæther et Schnell, 1988a

Chaudhuri et Sinharay, 1983

Wang et Sæther, 2001

Cranston et Saether, 1986

Halvorsen, 1982

Sæther, 1982

Sæther, 1983c

Sæther, 1992b

Wang et Sæther, 1993c

Sæther, 1985a

Hestenes et Sæther, 2000

Hestenes et Sæther, 2000

Sæther, 1981c

Sæther, 1981c

Hestenes et Sæther, 2000

Wen, Zhou et Rong, 1994

Sæther et Wang, 1992

Sæther, 1982

Schnell et Sæther, 1988

Oliver, 1985

Sæther, 1976

Sæther, 1976

Sæther, 1976

Sæther, 1976

Sæther, 1976

Andersen et Mendes, 2002a

Andersen et Sæther, 1996

Andersen et Sæther, 1996

Reiss et Sublette, 1985

Andersen et Sæther, 1996

Sæther, 1977a

Sæther et Andersen, 1998

Wang et al., 1993

Sæther, 1983a

$\begin{array}{ll}\text { HT, PT } & \mathrm{m}, \mathrm{f}, \mathrm{p} \\ \text { HT } & \mathrm{m} \\ \text { HT } & \mathrm{m}, \mathrm{f} \\ \text { HT } & \mathrm{m} \\ \text { HT } & \mathrm{m}, \mathrm{p} \\ \text { HT } & \mathrm{m} \\ \text { HT } & \mathrm{m} \\ \text { HT, PT } & \mathrm{m} \\ \text { HT } & \mathrm{m} \\ \text { HT } & \mathrm{m} \\ \text { HT } & \mathrm{m} \\ \text { HT } & \mathrm{m}, \mathrm{p} \\ \text { HT, PT } & \mathrm{m}, \mathrm{p}, \mathrm{l} \\ \text { HT, PT } & \mathrm{m}, \mathrm{p} \\ \text { HT } & \mathrm{m} \\ \text { HT, PT } & \mathrm{m}, \mathrm{f}, \mathrm{p}, 1\end{array}$

PT $m$

HT, PT m

HT $m$

HT m, f, p, 1

PT $\mathrm{f}$

PT $m$

PT $m$

PT $m$

PT $m$

HT, PT m, p

HT, PT m, f, p, 1

PT $m$

HT $m$

HT, PT m, f

HT, PT m, f

HT, PT m, f, p

HT $m$

HT m

PT $\mathrm{m}$

HT $m$

HT, PT m, f, l

HT, PT m, p

HT, PT m, f, p

HT, PT m, f, p

HT, PT m, f, p, l

PT $\mathrm{f}$

HT, PT m

HT, PT $m$

HT m, p

PT $m$

PT $\quad m, f$

PT $\quad \mathrm{m}, \mathrm{f}, \mathrm{p}, \mathrm{l}$

PT $\quad f, p$

PT $\quad \mathrm{m}, \mathrm{f}, \mathrm{p}, \mathrm{l}$

PT $\quad m, p$ 


$\begin{array}{ll} & \text { Microchironomus } \\ 311 & \text { Nilothauma } \\ 263 & \text { Nilothauma } \\ 261 & \text { Nilothauma } \\ 260 & \text { Nilothauma } \\ 257 & \text { Nilothauma } \\ 264 & \text { Nilothauma } \\ 258 & \text { Nilothauma } \\ 262 & \text { Nilothauma } \\ & \text { Nilothauma } \\ 265 & \text { Nilothauma } \\ 310 & \text { Nilothauma } \\ & \text { Nilothauma } \\ 259 & \text { Nilothauma } \\ 267 & \text { Paranilothauma } \\ & \text { Polypedilum } \\ & \text { Polypedilum } \\ & \text { Polypedilum } \\ 254 & \text { P. (Cerobregma) } \\ 255 & \text { P. (Cerobregma) } \\ 256 & \text { P. (Cerobregma) } \\ 371 & \text { P. (Tripodura) } \\ 366 & \text { P. (Tripodura) } \\ 365 & \text { P. (Tripodura) } \\ 372 & \text { P. (Tripodura) } \\ 369 & \text { P. (Tripodura) } \\ 370 & \text { P. (Tripodura) } \\ 367 & \text { P. (Tripodura) } \\ 368 & \text { P. (Tripodura) } \\ 275 & \text { P. (Uresipedilum) } \\ 278 & \text { P. (Uresipedilum) } \\ 274 & \text { P.(Uresipedilum) } \\ 277 & \text { P. (Uresipedilum) } \\ 273 & \text { P. (Uresipedilum) } \\ 276 & \text { P. (Uresipedilum) } \\ 272 & \text { P. (Uresipedilum) } \\ 271 & \text { P. (Uresipedilum) } \\ & \text { Robackia } \\ 85 & \text { Saetheria } \\ & \text { Xhangomyia } \\ & \text { Xiaostochironomironomus } \\ & \end{array}$

CHIRONOMINAE

247 Manoa

Pseudochironomus

Pseudochironomus

\section{CHIRONOMINAE}

Cladotanytarsus

Neostempellina

Parapsectra

317 Rheotanytarsus

clarilatus
acre
anderseni
ankasense
burmeisteri
duminola
flabellatum
fuscina
insolita
harrisoni
kakumense
mergae
sasai
verrucum
strebulosa
insolitum
nudiceps
obscurum
bulbocaudatum
subulatum
volselligum
akani
amplificatus
chaelum
dagombae
ewei
ogoouense
patulum
spinalveum
acutulum
anderseni
dossenudum
gladysae
harrisoni
kakumense
plautum
spinibojum
pilicauda
hirta
impectinata
aisenensis
laselvensis
aequipedes
plauta

\section{Pseudochironomini}

tangae

articaudus

badius

\section{Tanytarsini}

multispinulus

thienemanni

mendli

abonae

acuminatus

aquilus

atrius

baculus

beccus

buculicaudus
Guha et Chaudhuri, 1981

Adam et Sæther, 1999

Adam et Sæther, 1999

Adam et Sæther, 1999

Adam et Sæther, 1999

Adam et Sæther, 1999

Adam et Sæther, 1999

Adam et Sæther, 1999

Adam et Sæther, 1999

Adam et Sæther, 1999

Adam et Sæther, 1999

Adam et Sæther, 1999

Adam et Sæther, 1999

Adam et Sæther, 1999

Adam et Sæther, 2000

Chaudhuri et al., 1981

Chaudhuri et al., 1981

Chaudhuri et al., 1981

Sæther et Sundal, 1999

Sæther et Sundal, 1999

Sæther et Sundal, 1999

Bjørlo, 2002 in Vårdal et al. 2002

Bjørlo, 2002 in Vårdal et al. 2002

Vårdal, 2002 in Vårdal et al. 2002

Bjørlo, 2002 in Vårdal et al. 2002

Bjørlo, 2002 in Vårdal et al. 2002

Bjørlo, 2002 in Vårdal et al. 2002

Bjørlo, 2002 in Vårdal et al. 2002

Vårdal, 2002 in Vårdal et al. 2002

Oyewo et Sæther, 1998

Oyewo et Sæther, 1998

Oyewo et Sæther, 1998

Oyewo et Sæther, 1998

Oyewo et Sæther, 1998

Oyewo et Sæther, 1998

Oyewo et Sæther, 1998

Oyewo et Sæther, 1998

Sæther, 1977a

Sæther, 1983a

Sæther et Wang, 1993

Andersen et Kristoffersen, 1998

Andersen et Kristoffersen, 1998

Sæther et Wang, 1993

Sæther et Wang, 1993

Andersen et Sæther, 1997

Sæther, 1977a

Sæther, 1977a

Guha et al., 1985

Reiss, 1984a

Reiss, 1983

Kyerematen, 2000 in Kyerematen et al. 2000

Kyerematen et Sæthe,r 2000

Kyerematen et Sæther, 2000

Kyerematen et Sæther, 2000

Kyerematen et Andersen, 2002

Kyerematen et al., 2000

Kyerematen, 2000 in Kyerematen

$\begin{array}{ll}\text { HT, PT } & \mathrm{m}, \mathrm{f} \\ \text { PT } & \mathrm{m} \\ \text { PT } & \mathrm{m}\end{array}$

PT

HT

HT $m$

HT $\mathrm{m}$

HT, PT m

HT, PT $m$

HT, PT m

HT $m$

PT $\quad \mathrm{m}$

HT $m$

HT, PT m

PT $\quad m$

HT, PT m

HT $m$

PT $m$

PT $m$

PT $\mathrm{f}$

HT, PT m

HT $m$

HT, PT m, f

HT, PT $m$

HT, PT m

HT $m$

HT $m$

HT, PT m

HT $\mathrm{m}$

HT $\mathrm{m}$

HT, PT m

HT $m$

HT, PT m

HT, PT $\mathrm{m}$

HT, PT m

HT $m$

HT, PT m

HT, PT m

HT, PT m

PT $m$

HT f, p, 1

PT $f$

HT, PT m, f

HT $\mathrm{m}$

PT $\quad \mathrm{m}$

PT $m$

PT $m$

PT $m$

PT m

HT $\mathrm{m}$

HT, PT m

HT, PT $m$

HT, PT m

HT, PT $m$

HT $m$

HT, PT m
HT $m$ 
Rheotanytarsus

346 Rheotanytarsus

322 Rheotanytarsus

334 Rheotanytarsus

328 Rheotanytarsus

337 Rheotanytarsus

342 Rheotanytarsus

341 Rheotanytarsus

323 Rheotanytarsus

321 Rheotanytarsus

333 Rheotanytarsus

326 Rheotanytarsus

338 Rheotanytarsus

316 Rheotanytarsus

339 Rheotanytarsus

339 Rheotanytarsus Rheotanytarsus

330 Rheotanytarsus Rheotanytarsus

331 Rheotanytarsus

314 Rheotanytarsus

343 Rheotanytarsus

315 Rheotanytarsus

324 Rheotanytarsus

340 Rheotanytarsus

325 Rheotanytarsus

344 Rheotanytarsus

124 Rheotanytarsus

335 Rheotanytarsus

318 Rheotanytarsus

327 Rheotanytarsus

266 Seppia

Skutzia

353 Tanytarsus

364 Tanytarsus

Tanytarsus

Tanytarsus

304 Tanytarsus

351 Tanytarsus

Tanytarsus

Tanytarsus

Tanytarsus

305 Tanytarsus

306 Tanytarsus

307 Tanytarsus

308 Tanytarsus

125 Tanytarsus

309 Tanytarsus

Tanytarsus

352 Tanytarsus

Virgatanytarsus calakmulensis

contrerasi

digitatus

falcatus

falcipedus

foliatus

guanacastensis

hanseni

jongkindi

kjaeranderseni

koraensis

kuantanensis

kusii

longicornus

minusculus

nuamae

orientalis

pallidus

pantanalensis

phaselus

plerunguis

ramirezae

remus

sessilipersonatus

scutulatus

soelii

subtilis

thailandensis

thunesi

transversus

verticillus

trifurca

gaianii

ankasaensis

calorifontis

elisabethae

harei

kakumensis

mancospinosus

minimus

minutipalpus

monospinosus

pseudocongus

saetheri

spiesi

superpenicillatus

thaicus

tossai

tumultuarius

usambarae

ansatus et al. 2000

Kyerematen et Andersen, 2002

Andersen et Sæther, 2000 in

Kyerematen et al. 2000

Kyerematen et Sæther, 2000

Kyerematen et al., 2000

Kyerematen et al., 2000

Kyerematen et Andersen, 2002

Kyerematen et Andersen, 2002

Kyerematen et Andersen, 2002

Kyerematen et Sæther, 2000

Kyerematen et Sæther, 2000

Kyerematen et al., 2000

Kyerematen et al., 2000

Kyerematen et Andersen, 2002

Kyerematen et Sæther, 2000

Kyerematen, 2000 in Kyerematen et al. 2000

Kyerematen et Andersen, 2002

Moubayed, 1989

Kyerematen et al., 2000

Andersen et Kyerematen, 2001

Kyerematen et al., 2000

Kyerematen et Sæther, 2000

Kyerematen et Andersen, 2002

Kyerematen et Sæther, 2000

Kyerematen et al., 2000

Kyerematen et Andersen, 2002

Kyerematen et al., 2000

Kyerematen et Andersen, 2002

Moubayed, 1990

Kyerematen et Andersen, 2002

Kyerematen et Sæther, 2000

Kyerematen et al., 2000

Ekrem et Sæther 1999

Andersen, 2000

Ekrem, 2001

Ekrem, 2002

Ekrem, 2001

Ekrem, 2001

Ekrem, 1999

Ekrem et Reiss, 1999

in Ekrem et al. 1999

Guha et al., 1985

Ekrem et Harrison, 1999

Ekrem et Reiss, 1999

Ekrem, 1999

Ekrem, 1999

Ekrem, 1999

Ekrem, 1999

Moubayed, 1990

Ekrem, 1999

Ekrem et Reiss, 1999

Stur et Ekrem, 2000

Reiss, 1984b
PT $\quad \mathrm{m}$

HT, PT m, p

HT, PT $\mathrm{m}$

HT, PT m, f

HT, PT $m$

HT, PT m

HT, PT m

HT, PT m

HT $m$

HT, PT m, f, p, 1

HT, PT $m$

HT, PT m

HT $m$

HT f, p

HT m

HT $m$

HT $m$

HT, PT m

PT $m$

HT $m$

HT, PT m

HT, PT m, p

HT $m$

HT $m$

HT, PT m

HT $m$

HT, PT m

HT $m$

HT, PT m

HT, PT f, p

HT $m$

HT, PT m

PT $m$

HT $m$

HT, PT m, p

PT m, f, p, 1

PT $m$

HT $\mathrm{m}$

HT, PT m, f, p, 1

PT $m$

PT $\quad \mathrm{m}, \mathrm{f}$

PT $m$

HT, PT m

HT, PT m

HT $m$

HT, PT m

HT, PT m, p

HT, PT $m$

PT $m$

HT, PT m

PT $\mathrm{m}$ 


\section{Notes}

The following species has been synonymised or placed in other genera:

1) L. hudsoni Sæther, 1975 was synonymised with L. minimus (Meigen) by Sæther (1990).

2) L. nudiradius Sæther, 1975 was synonymised with $L$. natalensis (Kieffer) by Sæther (1990).

3) L. spatulosus Sæther, 1975 was synonymised with L. brachytomus (Kieffer) by Sæther (1990).

4) Phycoidella Sæther, 1971 is a junior synonym of Acamptocladius Brundin (Cranston \& Sæther 1982)

5) Rheosmittia halvorseni Cranston \& Sæther, 1986 belongs in Krenosmittia Thienemann \& Krüger (Tuiskunen \& Lideberg 1986).

6) A separate genus Onconeura Andersen et Sæther has been erected for $T$. semifimbriata Sæther, 1981 and new, related species (Andersen \& Sæther, in press).

7) Tokunagayusurika Sasa, 1978 is a junior synonym of Propsilocerus Kieffer (Sæther \& Wang 1996).

8) Oschia Sæther, 1983 is a junior synonym of Kloosia Kruseman (Cranston et al. 1989).

\section{Acknowledgement}

We are indebted to Prof. Ole A. Sæther for reading and correcting the present list repeatedly. Funding for compiling the list was given by the 'Det alminnelige naturvitenskapelige forskningsfond,' (The general natural science research fund), University of Bergen.

\section{References}

ADAM, J.I. \& SÆTHER, O.A. 1999. Revision of the genus Nilothauma Kieffer, 1921 (Diptera: Chironomidae). - Ent. scand., Suppl. 56: 1-107.

ADAM, J.I. \& SÆTHER, O.A. 2000. Paranilothauma strebulosa sp.n. from Costa Rica (Diptera: Chironomidae). Pp. 19-23 in: Hoffrichter, O. (ed.): Late 20th century research on Chironomidae: an anthology from the 13th international symposium on Chironomidae. Shaker Verl., Aachen, 661 pp.

ANDERSEN, T. 1996a. New Species of Diplosmittia Sæther, 1981 from Costa Rica (Chironomidae, Orthocladiinae). - Acta zool. Hung. 42: 127-132.

ANDERSEN, T. 1996b. A new species of Monodiamesa Kieffer, 1922 from southern Chile
(Diptera: Chironomidae: Prodiamesinae). - Rev. Chilena Ent. 23: 43-49.

ANDERSEN, T. 2000. A new species of Skutzia Reiss, 1985 (Chironomidae: Tanytarsini) from Henri Pittier National Park, Venezuela. - Bol. Entomol. Venez. 15: 119-125.

ANDERSEN, T.. \& CONTRERAS-RAmos, A. 1999. First record of Antillocladius Sæther from continental South America (Chironomidae, Orthocladiinae). - Acta zool. Hung. 45: 149-154.

ANDERSEN, T. \& KRISTOFFERSEN, L. 1998. New species of Xestochironomus Sublette and Wirth (Chironomidae: Chironominae) from Chile and Costa Rica. - J. Kansas ent. Soc. 71: 296-303.

ANDERSEN, T. \& KYEREMATEN, R.A.K. 2001. South American Rheotanytarsus Thienemann et Bause, with the description of one new species (Diptera: Chironomidae). - Norw. J. Entomol. 48: 269-274.

Andersen, T. \& MENDES, H.F. 2002a. New species and records of the Axarus "rogersigroup" from South and Central America (Diptera, Chironomidae). - Acta zool. Hung. 48: 79-84.

ANDERSEN, T. \& Mendes, H.F. 2002b. Neotropical and Mexican Mesosmittia Brundin, with the description of four new species (Insecta, Diptera, Chironomidae). - Spixiana 25: 55-69.

ANDERSEN, T. \& SCHNELL, Ø.A. 2000. New species of Bryophaenocladius Thienemann, 1934 from Tanzania, with bare squama (Diptera: Chironomidae). - Aquat. Insects 22: 48-57.

Andersen, T. \& SÆTher, O.A. 1993a. Colosmittia clavata gen.n., sp.n., a new orthoclad from the West Usambara Mountains, Tanzania (Diptera: Chironomidae). - J. Kansas ent. Soc. 66: 439443.

ANDERSEN, T. \& SÆTHER, O.A. 1993b. Lerheimia, a new genus of Orthocladiinae from Africa (Diptera, Chironomidae). - Spixiana 16: 105112.

ANDERSEN, T. \& SÆTHER, O.A. 1994a. Usambaromyia nigrala gen.n., sp.n., and Usambaromyiinae, a new subfamily among the Chironomidae (Diptera). - Aquat. Insects 16: 2129.

ANDERSEN, T. \& SÆTHER, O.A. 1994b. The first record of Buchonomyia Fittkau and the subfamily Buchonomyiinae from the New World (Diptera: Chironomidae). Pp. 363-367 in: P. Cranston (ed.): Chironomids: from genes to ecosystems. - CSIRO Australia, $482 \mathrm{pp}$.

ANDERSEN, T. \& SÆTHER, O.A. 1996. New species and records of Beardius Reiss et Sublette (Diptera: Chironomidae). - Annls Limnol. 32: 3344.

ANDERSEN, T. \& SÆTHER, O.A. 1997. First record of Manoa Fittkau and the tribe Pseudochironomini Sæther from the Afrotropical region (Diptera: Chironomidae: Chironomiae). Ent. scand. 28: 311-317.

ANDERSEN, T. \& SÆTHER, O.A. (in press). Onconeura, a new Neotropical orthoclad genus (Diptera: Chironomidae). - Mem. Inst. Oswaldo Cruz 00: 00-00. 
ANDERSEN, T. \& WANG, X. 1997. Darkwinged Heleniella Gowin, 1943 from Thailand and China (Insecta, Diptera, Chironomidae, Orthocladiinae). - Spixiana 20: 151-160.

ARMitAGE, P.D. 1985. A new species of the genus Limnophyes Eaton from S.E. England (Diptera, Chironomidae). In: Fittkau, E.J. (ed.): Beiträge zur Systematik der Chironomidae, Diptera. Spixiana, Suppl. 11: 139-142.

BRUNDIN, L. \& SÆTHER, O.A. 1978. Buchonomyia burmanica sp.n. and Buchonomyiinae, a new subfamily among the Chironomidae (Diptera). Zool. Scr. 7: 269-275.

Chaudhuri, P.K., DAS, S.K. \& Debnath, R.K. 1984. Studies of the Indian Tanypodinae (Diptera, Chironomidae) genus Tanypus Meigen. - Bull. Ent. Pologne 55: 99-109.

Chaudhuri, P.K., \& DeBNATH, R.K. 1984. A Coelotanypodine midge (Diptera: Chironomidae) from West Bengal, India. - Burdwan Univ. J. Sc. 1: 1-4.

Chaudhuri, P.K., Debnath, R.K. \& Nandi, S.K. 1983. Tanypodine midges of the genus Ablabesmyia Johannsen (Diptera: Chironomidae) from West Bengal with note on their seasonal incidence and sex ratios. - J. Nat. Hist. 17: 901917.

Chaudhuri, P.K. \& GHOSH, M. 1980. The Orthocladiinae (Diptera: Chironomidae) of India. Genus Cricotopus van der Wulp. - Aquat. Insects 2: 147-152.

Chaudhuri, P.K. \& GHOSH, M 1981. A new genus of podonomine midge (Chironomidae) from Bhutan. - Syst. Ent. 6: 373-376.

CHAUDHURI, P.K. \& GHOSH, M. 1982. Record of Chaetocladius Kieffer (Diptera: Chironomidae) from India. - Folia ent. Hung. 63: 5-7.

CHAUdHURI, P.K., GUHA, D.K. \& DAs GUPTA, S.K. 1981. Taxonomic studies of Chironominae (Diptera, Chironmidae) from India. The genus Polypedilum Kieffer. - Tijdschr. Ent. 124: 111147.

Chaudhuri, P.K. \& Sinharay, D.C. 1983. A study on Orthocladiinae (Diptera, Chironomidae) of India. The genus Rheocricotopus Thienemann and Harnisch. - Ent. Basiliensis 8: 398-407.

Chaudhuri, P.K. \& Sinharay, D.C. 1987. A study on Orthocladiinae (Diptera, Chironomidae) of India. Part V, genus Paraphaenocladius Thienemann. - Bull. Ent. 28: 95-103.

CRANSTON, P.S. \& EDWARD, D.H.D. 1999. Botryocladius gen.n.: a new transantarctic genus of orthocladiine midge (Diptera: Chironomidae). - Syst. Ent. 24: 305-333.

Cranston, P.S. \& Oliver, D.R. 1988. Aquatic xylophagous Orthocladiinae - systematics and ecology (Diptera: Chironomidae). - Spixiana 14: 143-154.

CRANSTON, P.S. \& SÆTher, O.A. 1982. A redefinition of Acamptocladius Brundin, 1956 (syn. Phycoidella Sæther, 1971, n.syn.) (Diptera: Chironomidae), with the description of $A$. reissi n.sp. - Ent. scand. 13: 25-32.

CRANSTON, P.S. \& SÆTHER, O.A. 1986. Rheosmittia (Diptera: Chironomidae): a generic validation and revision of the western Palaerctic species. - J. Nat. Hist. 20: 31-51.

EKREM, T. 1999. Six new Tanytarsus species from Ghana, West Africa (Insecta, Diptera, Chironomidae). - Spixiana 22: 53-68.

EKREM, T. 2001. A review of Afrotropical Tanytarsus van der Wulp (Diptera: Chironomidae). - Tijdschr. Ent. 144: 5-40.

EKREM, T. 2002. A review of selected South- and East Asian Tanytarsus v.d. Wulp (Diptera: Chironomidae). - Hydrobiologia 00: 00-00.

EKREM, T. \& HARRISON, A.D. 1999. Tanytarsus minutipalpus, spec. nov. from the saline lakes in the Rift Valley, East Africa (Insecta, Diptera, Chironomidae). - Spixiana 22: 199-208.

EKREM, T. \& REISS, F. 1999. Two new Tanytarsus species (Diptera: Chironomidae) from Brazil, with reduced median volsella. - Aquat. Insects 21: 1-9.

EKREM, T., REISS, F. \& LANGTON, P.H. 1999. Tanytarsus mancospinosus sp.n. (Diptera: Chironomidae) from eutrophic lakes in Europe. Norw. J. Entomol. 46: 79-87.

Ekrem, T. \& SÆTher, O.A. 1999. Seppia, a new Afrotropical tanytarsine genus (Diptera: Chironomidae). Pp. 79-87 in: HOFFRICHTER, O. (ed.): Late 20th century research on Chironomidae: an anthology from the 13th international symposium on Chironomidae. Shaker Verl., Aachen, 661 pp.

FERRINGTON, L.C., Jr. \& SÆTher, O.A. 1987. Male, female, pupa and biology of Oliveridia hugginsi n.sp. (Chironomidae: Diptera) from Kansas. - J. Kansas ent. Soc. 60: 451-461.

FERRINGTON, L.C., Jr. \& SÆTHER, O.A. 1994. Afrotropical species of Parakiefferiella Thienemann, with a review of species with palpal projections (Chironomidae: Diptera). Pp. 369-377 in: P. Cranston (ed.): Chironomids: from genes to ecosystems. - CSIRO Australia, $482 \mathrm{pp}$.

FITTKAU, E.J. \& MURRAY, D.A. 1983.

Pentaneurella katterjokki, eine neue Gattung und Art der Tanypodinae (Dipt. Chironomidae). Nachbl. Bayerischen Ent. 32: 57-63.

GHOSH, M. \& CHAUdHURI, P.K. 1983. Indian species of the genus Bryophaenocladius Thienemann (Diptera: Chironomidae). - J. Beng. nat. Hist. Soc. 2: 27-33.

GUHA, D.K. \& CHAUDHURI, P.K. 1981. Microchironomus clarilatus sp. nov. (Diptera, Chironomidae) from West Bengal, India. - Bull. zool. Surv. India 4: 143-145.

GUHA, D.K., DAS, S.K., CHAUDHURI, P.K. \& CHAUDHURI, D.K. 1985. Chironomid midges of the Andaman Islands (Diptera: Chironomidae). Proc. Natn. Acad. Sci. India 55: 22-38.

HALVORSEN, G.A. 1982. Saetheriella amplicristata gen.n., sp.n., a new Orthocladiinae (Diptera: Chironomidae) from Tennessee. - Aquat. Insects 4: 131-136.

Hestenes, T.C. \& S $Æ$ Ther, O.A. 2000. Three new Nearctic Thienemanniella Kieffer species with a review of the Nearctic species. Pp. 103-127 in: Hoffrichter, O. (ed.): Late 20th century research on Chironomidae: an anthology from the 13th 
international Symposium on Chironomidae. Shaker Verl., Aachen, 661 pp.

KYEREMATEN, R.A.K. \& ANDERSEN, T. 2002. Rheotanytarsus Thienemann et Bause (Diptera: Chironomidae) from Central America and Mexico. - Stud. Neotrop. Fauna Env. 37: 23-51.

KYEREMATEN, R.A.K., ANDERSEN, T. \& SÆTHER, O.A. 2000. A review of Oriental Rheotanytarsus Thienemann et Bause, with descriptions of some new species (Insecta, Diptera, Chironomidae). Spixiana 23: 225-258.

KYEREMATEN, R.A.K. \& SÆTHER, O.A. 2000. A review of Afrotropical Rheotanytarsus Thienemann et Bause, 1913 (Diptera: Chironomidae). - Tijdschr. Ent. 143: 27-69.

KYEREMATEN, R.A.K., SÆTHER, O.A. \& ANDERSEN, T. 2000. A review of the Rheotanytarsus pellucidus group (Diptera: Chironmidae). Pp. 147-170 in: Hoffrichter, O. (ed.): Late 20th century research on Chironomidae: an anthology from the 13th international symposium on Chironomidae. Shaker Verl., Aachen, 661 pp.

LANGTON, P. \& COBO, F. 1997. Metriocnemus (Inermipupa) carmencitabertarum subgen.n., sp.n. (Diptera: Chironomidae) from Spain and Portugal. - Entomologist's Gaz. 48: 263-271.

LANGTON, P.H. \& MOUBAYED, J. 2001. Limnophyes roquehautensis sp.n. and $L$. inanispatina sp.n. from southern France (Diptera, Chironomidae). - Nouv. Rev. Ent. 18: 3-8.

MoubAYED, Z. 1989. Descriptions of five new species of Chironomidae (Dipt., Chironomidae) from the Near East and the Oriental region. Acta biol. Debr. oecol. Hung. 2: 275-283.

MOUBAYED, Z. 1990. Chironomids from running waters of Thailand: description of Rheotanytarsus thailandensis sp.n. and Tanytarsus thaicus sp.n. (Dipt., Chironomidae). Hydrobiologia 203: 29-33.

OLIVER, D.R. 1977. Bicinctus-group of the genus Cricotopus Van der Wulp (Diptera: Chironomidae) in the Nearctic with a description of a new species. - J. Fish. Res. Bd Can. 34: 98104.

OLIVER, D.R. 1981. Description of Euryhapsis new genus including three new species (Diptera: Chironomidae). - Can. Ent. 113: 711-722.

OLIVER, D.R. 1984. Description of a new species of Cricotopus Van der Wulp (Diptera: Chironomidae) associated with Myriophyllum spicatum. - Can. Ent. 116: 1287-1292.

OLIVER, D.R. 1985. Review of Xylotopus Oliver and description of Irisobrillia n.gen. (Diptera: Chironomidae). - Can. Ent. 117: 1093-1110.

OLIVER, D.R \& Roussel, M.E. 1983. Redescription of Brillia Kieffer (Diptera: Chironomidae) with descriptions of Nearctic species. - Can. Ent. 115: 257-279.

OYEWO, E.A. \& SÆTHER, O.A. 1998. Revision of Afrotropical Polypedilum Kieffer subgen. Uresipedilum Sasa et Kikuchi, 1995 (Diptera: Chironomidae), with a review of the subgenus. Annls Limnol. 34: 315-362.
REISS, F. 1983. Parapsectra mendli n.sp. (Diptera, Chironomidae) aus dem Allgäu, Bayern. Spixiana 6: 79-81.

REISS, F. 1984a. Neostempellina thienemanni n.gen., n.sp., eine europäische Chironomide mit gehäusetragenden Larven (Diptera, Chironomidae). - Spixiana 7: 203-210.

REISS, F. 1984b. Virgatanytarsus ansatus n.sp. aus Mitteleuropa und Nordafrika (Diptera, Chironomidae). - Spixiana 7: 319-322.

ReISS, F. \& SubleTte, J.E. 1985. Beardius new genus with notes on additional Pan-American taxa (Diptera, Chironomidae). - Spixiana, Suppl. 11: 179-193.

SÆTHER, O.A. 1969. Some Nearctic Podonominae, Diamesinae, and Orthocladiinae (Diptera: Chironomidae). - Bull. Fish. Res. Bd Can. 170: $1-154$.

SÆTHER, O.A. 1971. Four new and unusual Chironomidae (Diptera). - Can. Ent. 103: 17991827.

SÆTHER, O.A 1973. Taxonomy and ecology of three new species of Monodiamesa Kieffer, with keys to Nearctic and Palaearctic species of the genus (Diptera: Chironomidae). - J. Fish. Res. Bd Can. 30: 665-679.

SÆTHER, O.A. 1975a. Two new species of Protanypus Kieffer, with keys to Nearctic and Palaearctic species of the genus (Diptera: Chironomidae). - J. Fish. Res. Bd Can. 32: 367388.

SÆTHER, O.A. 1975b. Two new species of Heterotanytarsus Spärck, with keys to Nearctic and Palaearctic males and pupae of the genus (Diptera: Chironomidae). - J. Fish. Res. Bd Can. 32: 259-270.

SÆTHER, O.A. 1975c. Nearctic and Palaearctic Heterotrissocladius (Diptera: Chironomidae). Bull. Fish. Res. Bd Can. 193: 1-67.

SÆTHER, O.A. 1975d. Twelve new species of the Limnophyes Eaton, with keys to Nearctic males of the genus (Diptera: Chironomidae). - Can. Ent. 107: 1029-1056.

SÆTHER, O.A 1976. Revision of Hydrobaenus, Trissocladius, Zalutschia, Paratrissocladius and some related genera (Diptera: Chironomidae). Bull. Fish. Res. Bd Can. 195: 1-287.

SÆTHER, O.A. 1977a. Taxonomic studies on Chironomidae: Nanocladius, Pseudochironomus and the Harnischia complex. - Bull. Fish. Res. Bd Can. 196: 1-143.

SÆTHER, O.A. 1977b. Habrobaenus hudsoni n.gen., n.sp. and the immatures of Baeoctenus bicolor Sæther (Diptera: Chironomidae). - J. Fish. Res. Bd Can. 34: 2354-2361.

SÆTHER, O.A. 1980. The females and immatures of Paracricotopus Thienemann and Harnisch, 1932, with the description of a new species (Diptera: Chironomidae). - Aquat. Insects 2: 129-145.

SÆTHER, O.A. 1981a. Compteromesa oconeensis gen. n., sp. n., a New Prodiamesinae (Diptera: Chironomidae) from South Carolina. - Aquat. Insects 3: 193-198.

SÆTHER, O.A. 1981b. Doncricotopus bicaudatus n.gen., n.sp. (Diptera: Chironomidae: 
Orthocladiinae) from the Northwest Territories, Canada. - Ent. scand. 12: 223-229.

SÆTHER, O.A. 1981c. Orthocladiinae (Diptera: Chironomidae) from the British West Indies with descriptions of Antillocladius n.gen., Lipurometriocnemus n.gen., Compterosmittia n.gen., and Diplosmittia n.gen. - Ent. scand., Suppl. 16: 1-46.

SÆTHER, O.A. 1982. Orthocladiinae (Diptera: Chironomidae) from SE U.S.A., with descriptions of Plhudsonia, Unniella and Platysmittia n.genera and Atelopodella n. subgen. - Ent. scand. 13: 465-510.

SÆTHER, O.A. 1983a. Oschia dorsenna n.gen., n.sp. and Saetheria hirta n.sp., two new members of the Harnischia complex (Diptera: Chironomidae). - Ent. scand. 24: 395-404.

SÆTHER, O.A. 1983b. Three new species of Lopescladius Oliveira, 1967 (syn. "Cordites" Brundin, 1966, n.syn.), with a phylogeny of the Parakiefferiella group. - Mem. Amer. Ent. Soc. 34: 279-298.

SÆTHER, O.A. 1983c. A review of Holarctic Gymnometriocnemus Goetghebuer, 1932, with the description of Raphidocladius subgen.n. and Sublettiella gen.n. (Diptera: Chironomidae). Aquat. Insects 5: 209-226.

SÆTHER, O.A. 1985a. Redefinition and review of Thienemannia Kieffer, 1909 (Diptera: Chironomidae), with the description of $T$. pilinucha sp.n. - Aquat. Insects 7: 111-131.

SÆTHER, O.A. 1985b. A review of Odontomesa Pagast, 1947 (Diptera: Chironomidae). Spixiana, Suppl. 11: 15-29.

SÆTHER, O.A. 1985c. Limnophyes er sp.n. (Diptera: Chironomidae, Orthocladiinae) from from Finland, with nearctic records of previously described species. - Ent. scand. 15: 540-544.

SÆTHER, O.A. 1985d. The imagines of Mesosmittia Brundin, 1956, with description of seven new species (Diptera, Chironomidae). - Spixiana, Suppl. 11: 37-54.

SÆTHER, O.A. 1985e. Diplosmittia carinata spec. nov. from Michigan (Diptera, Chironomidae). Spixiana, Suppl. 11: 55-57.

SÆTHER, O.A. 1985f. A review of the genus Rheocricotopus Thienemann \& Harnisch, 1932, with description of three new species (Diptera, Chironomidae). - Spixiana, Suppl. 11: 59-108.

SÆTHER, O.A. 1985g. Male and female imagines of Platysmittia bilyji sp. n. (Diptera: Chironomidae) from Manitoba, Canada. - Ent. scand. 15: 527 531.

SÆTHER, O.A. 1985h. Apometriocnemus fontinalis gen.n. sp.n. (Diptera: Chironomidae, Orthocladiinae) from Tennessee, U.S.A. - Ent. scand. 15: 536-539.

SÆTHER, O.A. 1985i. Heleniella parva sp.n. (Diptera: Chironomidae) from South Carolina and Tennessee, U.S.A. - Ent. scand. 15: 532535 .

SÆTHER, O.A. 1989a. Metriocnemus van der Wulp: a new species and a revision of species described by Meigen, Zetterstedt, Staeger, Holmgren, Lundstöm and Strenzke (Diptera: Chironomidae). - Ent. scand. 19: 393-430.
SÆTHER, O.A. 1989b. Two new species of Hydrobaenus Fries from Massachusetts, U.S.A. and Japan (Diptera: Chironomidae). - Ent. scand. 20: 55-63.

SÆTHER, O.A. 1990. A review of the genus Limnophyes Eaton from the Holarctic and Afrotropical regions (Diptera: Chironomidae, Orthocladiinae). - Ent. scand., Suppl. 35: 1-139.

SÆTHER, O.A. 1992a. First Palaearctic record of the orthoclad Plhudsonia Sæther (Diptera: Chironomidae). - Ent. scand. 22: 379-384.

SÆTHER, O.A. 1992b. First Nearctic record of the orthoclad genus Tavastia Tuiskunen (Diptera: Chironomidae). - Ent. scand. 22: 385-388.

SÆTHER, O.A. 1992c. Heterotrissocladius boltoni sp.n., a new orthoclad from vernal pools and streams in Ohio, U.S.A. (Diptera: Chironomidae). - Neth. J. Aquat. Ecol. 26: 191196.

SÆTHER, O.A. 1995. Metriocnemus van der Wulp: seven new species, revision of species, and new records (Diptera: Chironomidae). - Annls limnol. 31: 35-64.

SÆTHER, O.A. 1996. Afrotropical records of the orthoclad genus Mesosmittia Brundin (Insecta, Diptera, Chironomidae). - Spixiana, Suppl. 11: 37-54.

SÆTHER, O.A. \& ANDERSEN, T. 1993. Lobosmittia, a new genus of orthoclads from Tanzania and Turkey (Diptera: Chironomidae). - Tijdschr. Ent. 136: 283-287.

SÆTHER, O.A. \& ANDERSEN, T. 1995. Ionthosmittia caudiga n.gen. n.sp., a new orthoclad from the Usambara Mts, Tanzania (Diptera, Chironomidae). - Trop. Zool. 8: 197-202.

SÆTHER, O.A. \& ANDERSEN, T. 1996. First Afrotropical records of Doithrix and Georthocladius, with notes on the Pseudorthocladius group (Diptera: Chironomidae). - Tijdschr. Ent. 139: 243-256.

SÆTHER, O.A. \& ANDERSEN, T. 1998. Friederia, a new Afrotropical tanytarsine genus (Diptera: Chironomidae). - Ent. scand. 29: 29-37.

SÆTHER, O.A. \& ANDERSEn, T. 1999. Molleriella, a new terrestrial orthoclad genus from the Netherlands (Diptera: Chironomidae). - Acta zool. Hung. 45: 161-168.

SÆTHER, O.A. \& SCHNELl, Ø.A. 1988a. Two new species of the Rheocricotopus (R.) effusus group (Diptera, Chironomidae). - Spixiana, Suppl. 14: 65-74.

SÆTHER, O.A. \& SCHNELL, Ø.A. 1988 b. Heterotrissocladius brundini spec. nov. from Norway (Diptera, Chironomidae). - Spixiana, Suppl. 14: 57-64.

SÆTHER, O.A. \& SUBLETTE, J. 1983. A review of the genera Doitrix n.gen., Georthocladius Strenzke, Parachaetocladius Wülker and Pseudorthocladius Goetghebuer (Diptera: Chironomidae, Orthocladiinae). - Ent. scand., Suppl. 20: 1-100.

SÆTHER, O.A. \& SundAL, A. 1999. Cerobregma, a new subgenus of Polypedilum Kieffer, with a tentative phylogeny of subgenera and species groups within Polypedilum (Diptera: 
Chironomidae). - J. Kansas ent. Soc. 71: 315382.

SÆTHER, O.A. \& WANG, X. 1993. Xiaomyia, Shangomyia and Zhouomyia, three new and unusual genera of Chironomini from Oriental China (Diptera: Chinomidae). - Ent. scand. 24: 185-195.

SÆTHER, O.A. \& WANG, X. 1995. Revision of the genus Paraphaenocladius Thienemann, 1924 of the world (Diptera: Chironomidae, Orthocladiinae). - Ent. scand., Suppl. 48: 1-69.

SÆTHER, O.A. \& WANG, X. 1996. Revision of the orthoclad genus Propsilocerus Kieffer ( = Tokunagayusurika Sasa) (Diptera: Chironomidae). - Ent. scand. 27: 441-479.

SÆTHER, O.A. \& WiLlassen, E. 1987. Four new species of Diamesa Meigen, 1835 (Diptera: Chironomidae) from the glaciers of Nepal. - Ent. scand., Suppl. 29: 189-203.

SÆTHER, O.A. \& WILlASSEN, E. 1988. A review of Lappodiamesa Serra Tosio, with the description of L. boltoni from Ohio, U.S.A. (Diptera: Chironomidae). - Spixiana, Suppl. 14: 75-84.

SCHNELL, Ø.A. 1991. A new species of Bryophaenocladius Thienemann (Diptera: Chironomidae) from Norway with emendations to the diagnoses of the genus. - Ent. scand. 21: 435-443.

SCHNELL, Ø.A. \& SÆTHER, O.A. 1988. Vivacricotopus, a new genus of Orthocladiinae from Norway (Diptera, Chironomidae). Spixiana, Suppl. 14: 49-55.

SOPONIS, A.R. 1990. A revision of the Holarctic species of Orthocladius (Euorthocladius) (Diptera: Chironomidae). - Spixiana, Suppl. 13: $1-56$.

STUR, E. \& ANDERSEN, T. 2000. A new Physoneura Ferrington et Sæther, 1995, from Ecuador (Chironomidae, Orthocladiinae). Norw. J. Entomol. 47: 131-136.

STUR, E. \& EKREM, T. 2000. Tanytarsus usambarae, spec. nov. from West Usambara Mts., Tanzania, East Africa. - Spixiana 23: 219223.

TUISKUNEN, J. \& LINDEBERG, B. 1986. Chironomidae (Diptera) from Fennoscandia north of $68^{\circ} \mathrm{N}$, with a description of ten new species and two new genera. - Ann. zool. fenn. 23: 361-393.

VÅRDAL, H., BJøRLO, A. \& SÆTHER, O.A. 2002. Afrotropical Polypedilum subgenus Tripodura, with a review of the subgenus (Diptera: Chironomidae). - Zool. Scr. 31: 00-00.

WANG, X. 1998. Compterosmittia virga, a new species from China (Diptera: Chironomidae). Acta ent. sin. 41: 95-97.

WANG, X. \& HALVORSEN, G.A. 2002. A new Eukiefferiella Thienemann, 1926 from Northeast
China (Diptera, Chironomidae). - Aquat. Insects 24: 123-128.

WANG, X. \& SÆTHER, O.A. 1992. Euryhapsis fuscipropes sp.n. from China and Tokyobrillia anderseni sp.n. from Tanzania, with a review of genera near Irisobrillia Oliver (Diptera Chironomidae). - Annls Limnol. 28: 209-223.

WANG, X. \& SÆTher, O.A. 1993a. First Palaearctic and Oriental records of the orthoclad genus Antillocladius Sæther (Diptera: Chironomidae). Ent. scand. 24: 227-230.

WANG, X. \& SÆTHER, O.A. 1993b. Limnophyes Eaton from the China, with the description of five new species (Diptera: Chironomidae). - Ent. scand. 24: 215-226.

WANG, X. \& SÆTHER, O.A. 1993c. A new species of the 'marine' genus Thalassosmittia Strenzke \& Remmert from Xizang (Tibet), China (Diptera: Chironomidae). - Ent. scand. 24: 211-214.

WANG, X. \& SÆThER, O.A. 1998. Quiniella, a new orthoclad genus from China (Diptera: Chironomidae). - Hydrobiologia 362: 103-106.

WANG, X. \& SÆTHER, O.A. 2001. Two new species of the orientalis group of Rheocricotopus (Psilocricotopus) from China (Diptera: Chironomidae). - Hydrobiologia 444: 237-240.

WANG, X. \& SÆTHER, O.A. 2002. Hanocladius, a new orthoclad genus from China (Diptera: Chironomidae). - Hydrobiologia 468: 181-183.

WANG, X. , SÆThER, O.A. \& ANDERSEN, T. 2001. Afrotropical Bryophaenocladius Thienemann, 1934 (Diptera: Chironomidae). - Stud. dipt. 8: 447-462.

WANG, X., ZHENG, L. \& Ji, B. 1993. A taxonomic study on Chironominae from China III. Genus Harnischia Kieffer. - Acta zootaxon. sin. 18: 459-465

WANG, X., ZHENG, L. \& Ji, B. 1994. A taxonomic study on Orthocladiinae (Dipterta: Chironomidae) of China: II. Genus Brillia Kieffer. - Acta ent. sin. 37: 359-363.

Wen, T., ZHOU, C. \& RoNG, Y. 1994. Description of the new species Togunagayusurika taihuensis (Diptera: Chironomidae). - Entomotaxonomia 16: 205-212. (In chinese with English summary.)

WIDERHOLM, T. 1975. Description of Protanypus saetheri n.sp. from Alaska (Diptera: Chironomidae). - Ent. scand. 6: 224-228.

WILlASSEN, E. 1985. A review of Diamesa davisi Edwards and the davisi group. - Spixiana, Suppl. 11: 109-137.

WILLASSEN, E. 1996. A nival Bryophaenocladius Thienemann, 1934, with reduced wings (Insecta: Diptera: Chironomidae). - Ann. Naturhist. Mus. Wien 98: 507-512.

WILlASSEN, E. \& CRANSTON, P.S. 1986. Afrotropical montane midges (Diptera, Chironomidae, Diamesa). - Zool. J. Linn. Soc. 87: 91-123. 